\title{
A Rare Case of Calf Muscle Metastasis from a Non-Functional Pancreatic Neuroendocrine Carcinoma
}

\author{
Li Na Shi ${ }^{1, *}$; Zhong Ling Qiu ${ }^{2}$; Chun Gen Wu ${ }^{1}$; Quan Yong Luo ${ }^{2}$ \\ ${ }^{1}$ Department of Radiology, Shanghai Sixth People's Hospital, Shanghai Jiao Tong University, Shanghai, China \\ ${ }^{2}$ Department of Nuclear Medicine, Shanghai Sixth People's Hospital, Shanghai Jiao Tong University, Shanghai, China \\ *Corresponding author: Li Na Shi, Department of Radiology, Shanghai Sixth People's Hospital, Shanghai Jiao Tong University, Shanghai 200233, China. Tel: +86-2164844183, Fax: +86- \\ 2164844183, E-mail: slnssmu2008@sina.com
}

Received: April 17, 2013; Revised: June 23, 2013; Accepted: July 23, 2013

\begin{abstract}
Pancreatic neuroendocrine tumors (PNET) are uncommon pancreatic neoplasms, accounting for 1-2\% of all pancreatic tumors. However, they have a better prognosis and long-term survival compared to exocrine pancreatic cancer. PNETs can be divided into functional or nonfunctional based upon whether or not they excrete active substances relevant to specific clinical syndromes. Skeletal muscle metastasis is also a rare condition and differentiation between a primary soft tissue sarcoma and metastatic carcinoma is difficult without biopsy. Thus, skeletal muscle metastases from pancreatic neoplasms are exceedingly rare, with only a few cases reported in the literature. We present a 34-year-old man with metastatic pancreatic neuroendocrine carcinoma that was initially thought to be a primary soft tissue tumor. Pathology and immunohistochemistry demonstrated the tumor to be a metastasis from a pancreatic neuroendocrine carcinoma. A brief review of the literature on this subject is also presented.
\end{abstract}

Keywords:Pancreatic Neoplasms; Neuroendocrine Carcinoma; Metastasis

\section{Introduction}

The usual sites of metastases in pancreatic cancer are the liver and peritoneal cavity. Other less common sites are the lung, bone, and brain (1). Unusual sites such as muscle, skin, heart, pleura, stomach, umbilicus, kidney, appendix, spermatic cord, and prostate have also been reported (2-4). Skeletal muscle metastases from pancreatic cancer have by far been considered as an infrequent occurrence. Although the true incidence of skeletal muscle metastases in patients with pancreatic cancer is not known, skeletal muscle metastases from pancreatic carcinoma are exceedingly rare with only a few cases reported in the literature. We present a 34-year-old man with left calf pain, tumescence, and transient epigastric pain for 3 months. In CT and MRI, this appeared to be a primary soft tissue tumor. However, calf muscle biopsy demonstrated metastatic pancreatic neuroendocrine carcinoma.

\section{Case Presentation}

A 34-year-old man of Asian descent presented with a 3 month history of pain and tumescence in the left calf. He also complained of occasional and transient vague epigastric pain, without fever, nausea, or vomiting. There was no family history of gastrointestinal cancer. On physical examination, the left calf was swollen, with a large $(8 \mathrm{~cm})$ palpable lobulated and firm mass with an ill-defined margin. Abdominal examination was unre- markable: nontender and without any masses. Incidentally, his CA-125 level was $82.77 \mathrm{U} / \mathrm{mL}$ (reference range: 0-35 U/mL). Left calf plain films demonstrated a large soft tissue mass behind the tibia and fibula without a clear border but confined to the soft tissue without bony abnormality (Figure 1). Computerized tomography (CT) showed a heterogeneous mass with multiple fine compartments (Figure 2). The mass was confined to the soft tissue and did not involve the adjacent bone. Magnetic resonance imaging (MRI) displayed heterogeneous signal intensity on T1-weighted images and fat-suppression T2-weighted images (Figure $3 \mathrm{~A}, \mathrm{~B}$ ) with obvious intensification (Figure 4). All these radiological characteristics suggested a primary soft tissue tumor. The patient underwent fine needle aspiration and biopsy. Pathology demonstrated metastatic neuroendocrine carcinoma (Figure 5 A-C). Subsequently, the patient underwent contrast CT of the abdomen, which showed a large, irregular, low-density mass in the head, body, and tail of the pancreas without obvious enhancement (Figure $6 \mathrm{~A}, \mathrm{~B})$. Besides, there was another hypodense mass in the medial limb of the right adrenal. The patient underwent a pancreaticoduodenectomy and pathology confirmed the diagnosis. Regional lymph nodes were positive for a poorly-differentiated neuroendocrine carcinoma. The mass located in the right adrenal was also

Copyright ( ) 2015, Tehran University of Medical Sciences and Iranian Society of Radiology. This is an open-access article distributed under the terms of the Creative Commons Attribution-NonCommercial 4.0 International License (http://creativecommons.org/licenses/by-nc/4.0/) which permits copy and redistribute the material just in noncommercial usages, provided the original work is properly cited. 


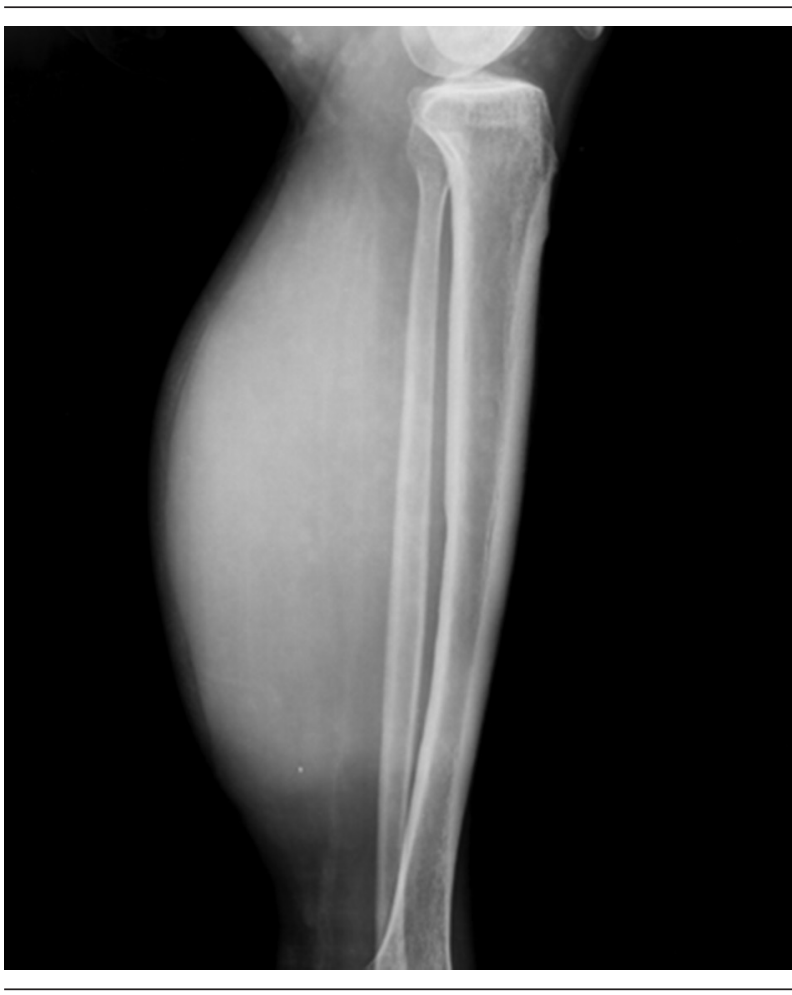

Figure 1. A 34-year-old man with a 3-month history of pain and tumescence in the left calf. Lateral plain radiography of the left calf, demonstrat

ing a large soft tissue mass behind the tibia and fibula
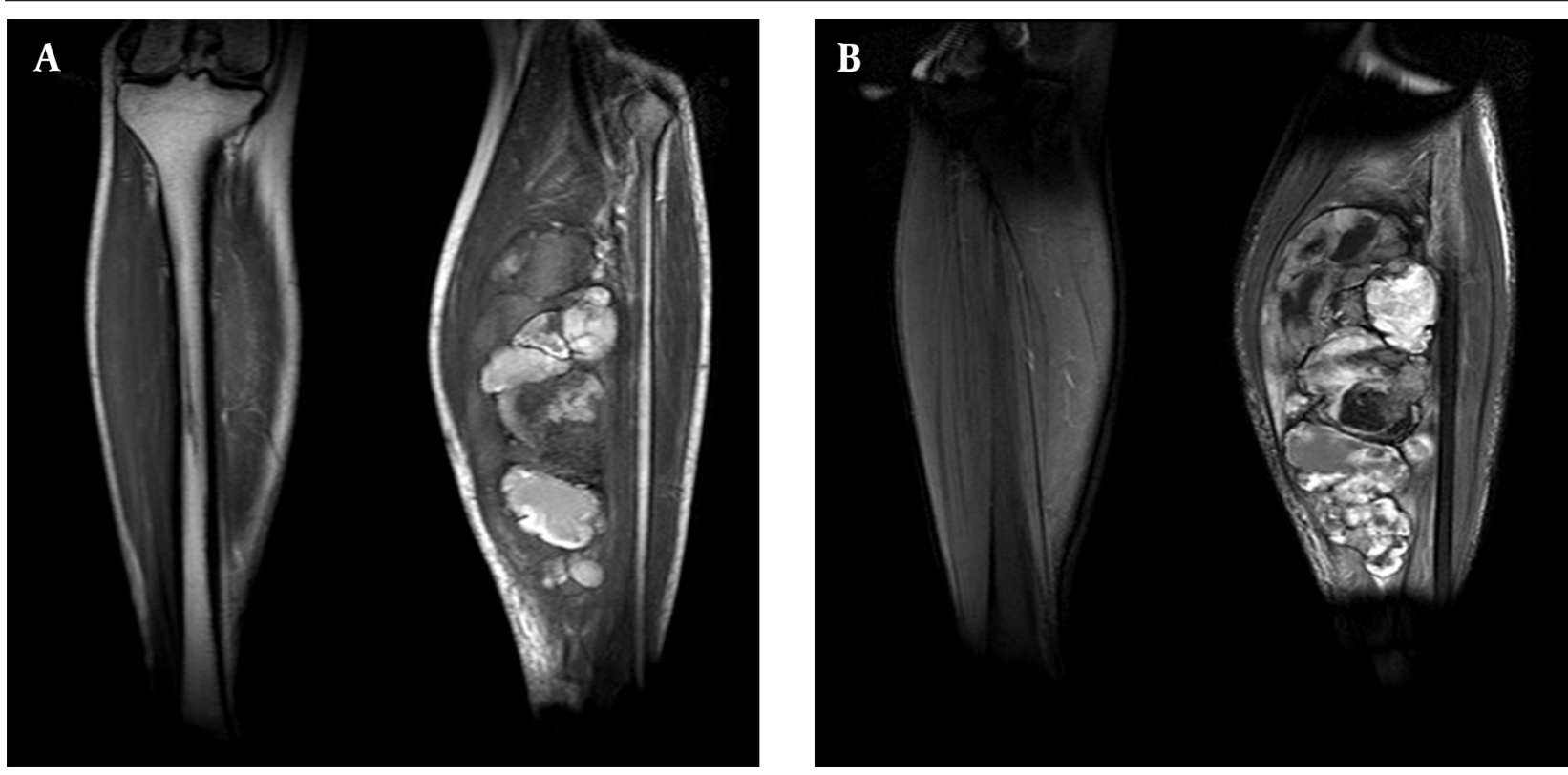

Figure 3. (A and B), Magnetic resonance imaging (MRI) demonstrating heterogeneous signal intensity in the muscle on T1-weighted and fat-suppression T2-weighted images resected and confirmed as adenoma, which was unrelated to the lesion in the pancreas. He also underwent below the knee amputation and pathology confirmed

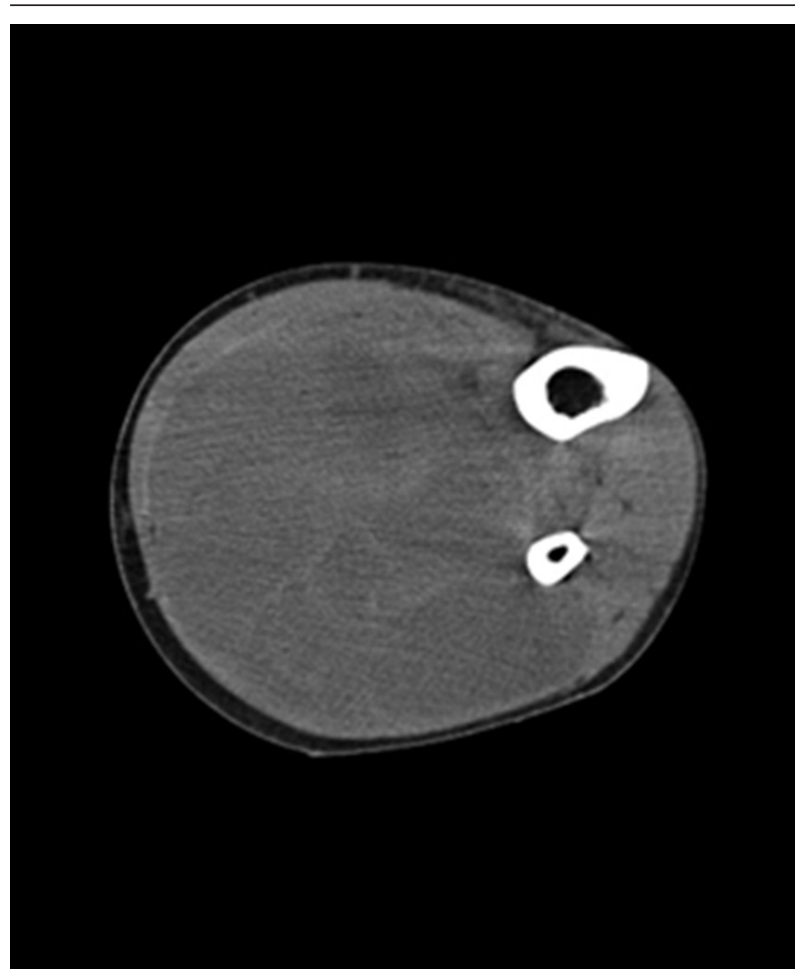

Figure 2. Computerized tomography of the left calf, demonstrating a het erogeneous mass with fine compartments 


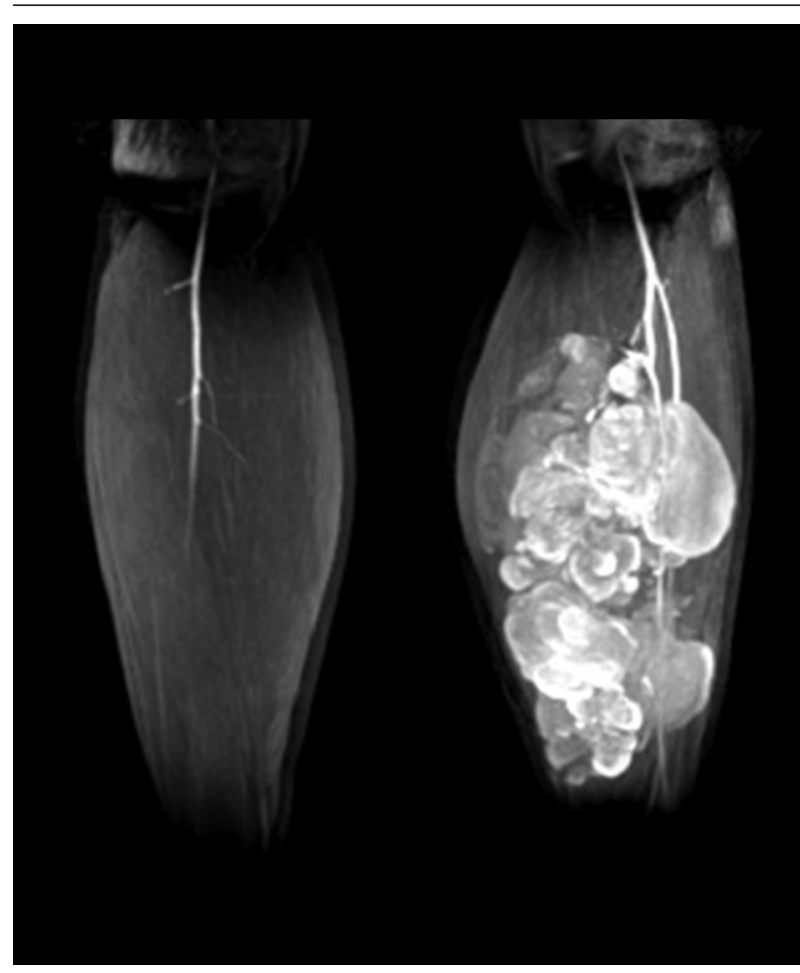

Figure 4. Contrast-enhanced magnetic resonance angiography with obvious enhancement and "cluster of grapes" appearance

\section{Discussion}

Pancreatic neuroendocrine tumors (PNET), a subgroup of gastroenteropancreatic neuroendocrine tumors, have an incidence of $1 / 100,000$ and represent $1-2 \%$ of all pancreatic neoplasms $(5,6)$. PNETs are classified as functional or non-functional based upon the presence or absence of a specific clinical syndrome relevant to hormone oversecretion. Recently, a higher number of nonfunctional pancreatic neuroendocrine tumors have been detected coinciding with the increased use of cross-sectional imaging. The overall prognosis and long-term survival in PNET are far better than in patients with exocrine pancreatic cancer (7), which has one of the highest mortality rates of any cancer with a 5-year survival of less than 5\% (8). Pancreatic cancer is known to metastasize rapidly. Liver and peritoneum are the most common sites of metastases in pancreatic cancer, followed by the lungs, bone, and brain (1). Skeletal muscle metastasis is a rare condition (9) and differentiation between a primary soft tissue sarcoma and metastatic carcinoma is difficult without biopsy (10). Skeletal muscle metastases from pancreatic carcinoma are extremely infrequent. To the best of our knowledge, there have been only a few cases of skeletal muscle metastases from pancreatic carcinoma reported in the English literature. Previously reported involved muscles are the gluteal muscle, psoas, rectus abdominis, pectoralis major, quadriceps femoris, and biceps
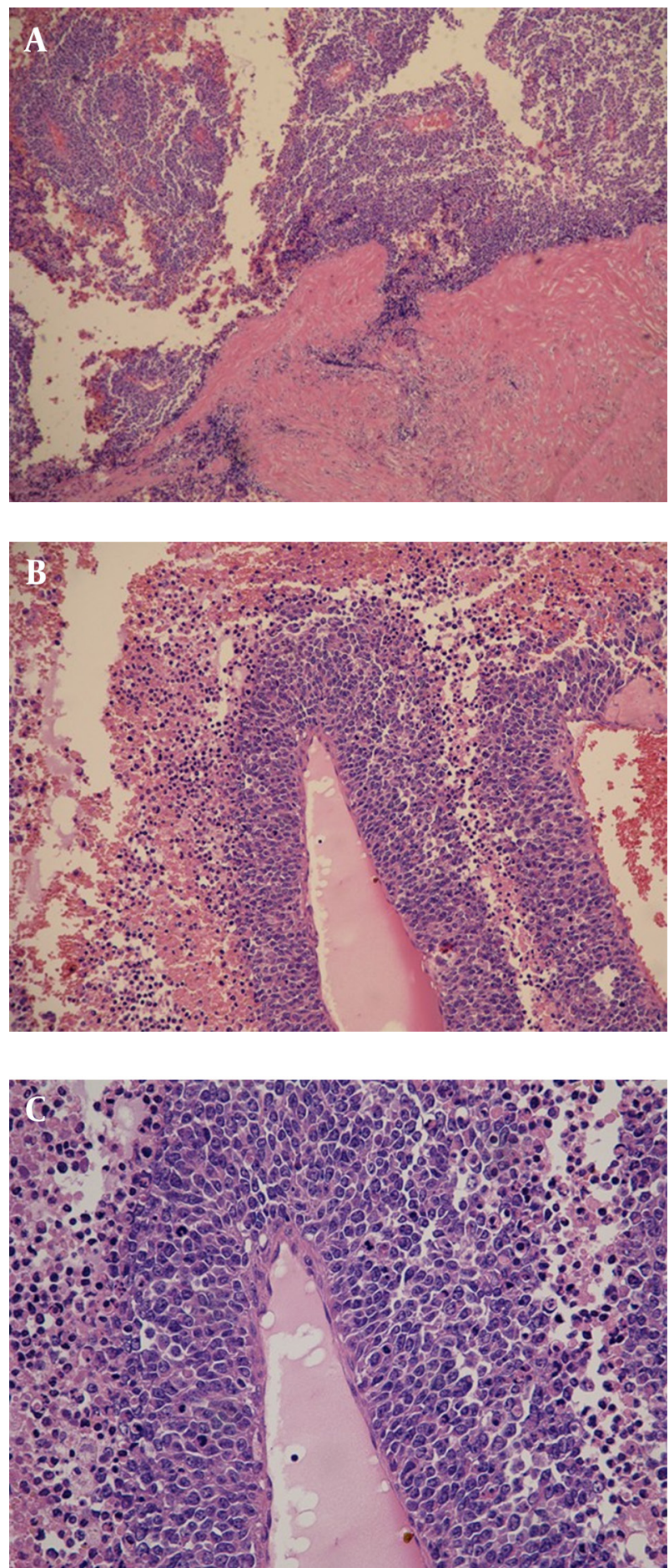

Figure 5. (A, B and C), Microscopic appearance of calf metastasis from pancreatic neuroendocrine carcinoma $(\mathrm{H} \& \mathrm{E}, 40 \times, 100 \times, 400 \times)$

(Table 1) (11-13). Metastasis to the triceps surae has not been mentioned. Patients with lung and thyroid cancer, melanoma and lymphoma may present with skeletal muscle metastasis, but pancreatic carcinoma is a particularly unusual primary malignancy for this finding (14). 

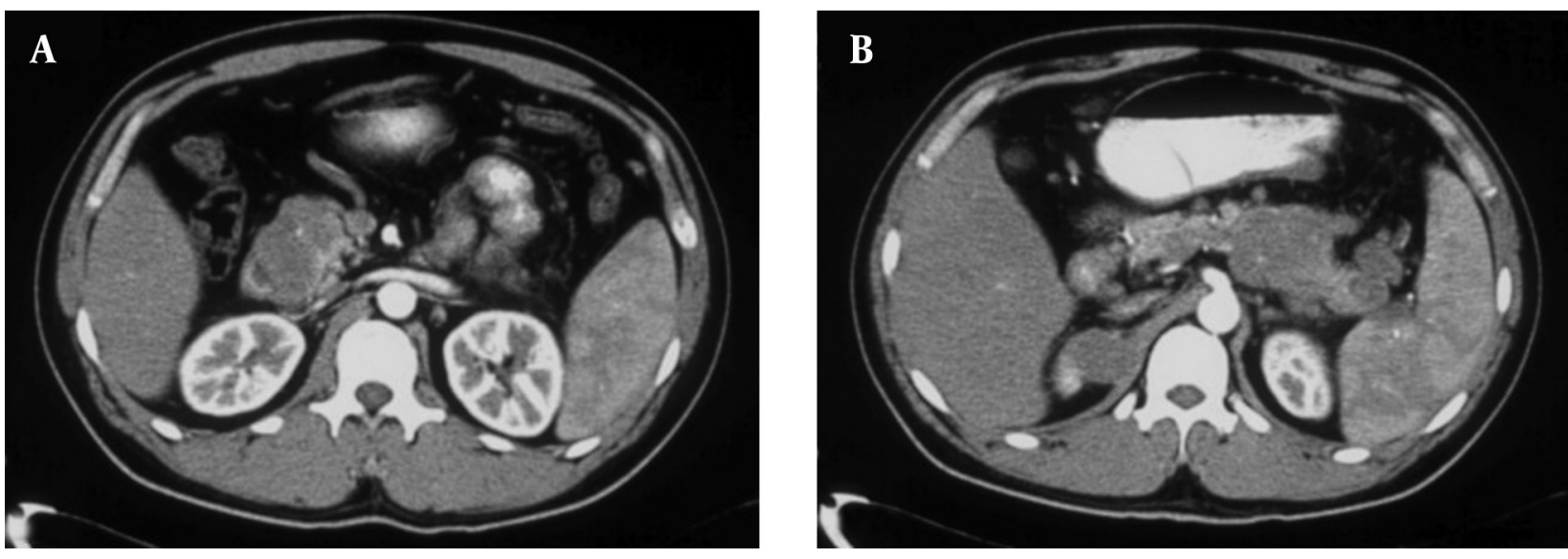

Figure 6. (A and B), Contrast CT scan of the abdomen showing a low-density mass in the head, body, and tail of the pancreas without obvious enhancement.

Table 1. Reported Cases of Muscular Metastasis from Pancreatic Neoplasms

\begin{tabular}{lccc}
\hline Author & Year & Number of Case & Metastatic Site \\
\hline Larson et al.(11) & 1988 & 6 & $\begin{array}{c}\text { Psoas, Rectus abdominis, Pectoralis major, Quadriceps femoris, } \\
\text { Biceps, Gluteus maximus }\end{array}$ \\
Sironi et al.(13) & 1991 & 1 & Right thigh \\
Agzarian et al.(12) & 2007 & 1 & Gluteal muscle (bilateral) \\
\hline
\end{tabular}

Although skeletal muscle comprises nearly $50 \%$ of the total body weight, and has an abundant blood supply, metastasis to the skeletal muscle is an infrequent phenomenon $(14,15)$. The mechanism of skeletal muscle metastasis in patients with pancreatic cancer is not well known, but it may be related to turbulent blood flow, high tissue pressure, contractility, accumulation of lactic acid, $\mathrm{pH}$ environment, and oxygenation $(9,16)$. Diagnosis is confirmed mostly by needle aspiration. However, before pathological diagnosis, radiological assessment of the mass is necessary and often provides useful information with respect to the overall assessment and treatment of this lesion. In comparison with the MR image, plain film and $\mathrm{CT}$ are of little value regarding the characterization of the lesions. MRI can be used not only for diagnosis of metastatic tumors, sarcomas, hematomas, and abscesses, but also for delineating the extent of the involved muscles from adjacent muscles on multiplanar sections $(17,18)$ and accurately determining invasion to the bone and vascular structures. Besides, MR appearance is also helpful in planning the treatment strategy. In particular, contrast-enhanced MRI helps in evaluating the blood supply of the tumor in designing the excision plan of the lesion. In a word, although MR imaging is not a specific imaging tool for skeletal muscle metastasis (19), it is an essential imaging modality for the diagnosis and treatment of soft tissue tumors. As demonstrated by this case, biopsy with histology and immunohistochemistry should be performed for the final diagnosis of the mass and to differentiate primary tumors from metastases. Our CT and MRI findings favored a primary soft tissue tumor. A tissue diagnosis is especially useful in the case of nonspecific imaging findings or atypical clinical features. Skeletal muscle metastases from pancreatic cancer and other malignant tumors do occur and should be considered in cancer patients with soft tissue abnormalities and pain. Biopsy with accompanying histology and immunohistochemistry play a vital role in identifying primary tumor from metastatic disease.

\section{Authors' Contributions}

Zhong-Ling Qiu developed the original idea and the protocol. Li-Na Shi abstracted and analyzed the data, wrote and revised the manuscript, and is the first and corresponding author. Quan-Yong Luo and Chun-Gen Wu contributed to the development of the protocol.

\section{References}

1. Borad MJ, Saadati H, Lakshmipathy A, Campbell E, Hopper P, Jameson G, et al. Skeletal metastases in pancreatic cancer: a retrospective study and review of the literature. Yale J Biol Med. 2009;82(1):1-6.

2. Otegbayo JA, Oluwasola OA, Akere A, Yakubu A, Daramola OO, Ogun GO. Pancreatic carcinoma presenting as cutaneous nodules in a diabetic Nigerian male. West Afr J Med. 2005;24(2):180.

3. Crescentini F, Deutsch F, Sobrado CW, Araujo S. Umbilical mass as the sole presenting symptom of pancreatic cancer: a case report. Rev Hosp Clin Fac Med Sao Paulo. 2004;59(4):198-202.

4. Martino L, Martino F, Coluccio A, Mangiarini MG, Chioda C. Re- 
nal metastasis from pancreatic adenocarcinoma. Arch Ital Urol Androl. 2004;76(1):37-9.

5. Metz DC, Jensen RT. Gastrointestinal neuroendocrine tumors: pancreatic endocrine tumors. Gastroenterology. 2008; 135(5):1469-92.

6. Oberg K, Eriksson B. Endocrine tumours of the pancreas. Best Pract Res Clin Gastroenterol. 2005;19(5):753-81.

7. Fesinmeyer MD, Austin MA, Li CI, De Roos AJ, Bowen DJ. Differences in survival by histologic type of pancreatic cancer. Cancer Epidemiol Biomarkers Prev. 2005;14(7):1766-73.

8. Kumar V, Abbas AK, Fausto N. Robbins and Cotran Pathologic Basis of Disease. 7th edPhiladelphia: Elsevier Saunders; 2005.

9. Seely S. Possible reasons for the high resistance of muscle to cancer. Med Hypotheses. 1980;6(2):133-7.

10. Herring CJ, Harrelson JM, Scully SP. Metastatic carcinoma to skeletal muscle. A report of 15 patients. Clin Orthop Relat Res. 1998(355):272-81.

11. Larson DA, Bottles K, Federle M, Fippin L, Luce J. Skeletal muscle metastases from pancreatic cancer. Onkologie.1988;11(6):282-5.

12. Agzarian MJ, Jones DN, Yong TY, Roberts-Thomson P. Bilatera symmetric gluteal muscle metastases from pancreatic carcinoma presenting as a myositis. Australas Radiol. 2007;51 Spec
No.:B119-21.

13. Sironi M, Radice F, Taccagni GL, Braga M, Zerbi M. Fine needle aspiration of a pancreatic oxyphilic carcinoma with pulmonary and subcutaneous metastases. Cytopathology.1991;2(6):303-9.

14. Tuoheti Y, Okada K, Osanai T, Nishida J, Ehara S, Hashimoto M, et al. Skeletal muscle metastases of carcinoma: a clinicopathological study of 12 cases. Jpn J Clin Oncol. 2004;34(4):210-4.

15. Hundt W, Braunschweig R, Reiser M. Diffuse metastatic infiltration of a carcinoma into skeletal muscle. Eur Radiol. 1999;9(2):208-10.

16. Michalaki V, Zygogianni A, Kouloulias V, Balafouta M, Vlachodimitropoulos D, Gennatas CG. Muscle metastasis from hepatocellular carcinoma. J Cancer Res Ther. 2011;7(1):81-3.

17. Sudo A, Ogihara Y, Shiokawa Y, Fujinami S, Sekiguchi S. Intramuscular metastasis of carcinoma. Clin Orthop Relat Res. 1993(296):213-7.

18. Williams JB, Youngberg RA, Bui-Mansfield LT, Pitcher JD. MR imaging of skeletal muscle metastases. AJR Am J Roentgenol. 1997;168(2):555-7.

19. Kransdorf MJ, Jelinek JS, Moser RJ, Utz JA, Brower AC, Hudson TM, et al. Soft-tissue masses: diagnosis using MR imaging. AJR Am J Roentgenol.1989;153(3):541-7. 